\section{Lyapunov-Based Range Identification For Paracatadioptric Systems}

Guoqiang Hu, Darren Aiken, Sumit Gupta, and Warren. E. Dixon

\begin{abstract}
Many applications require the interpretation of the Euclidean coordinates of features of a 3-D object through 2-D images. In this paper, the relative range and the Euclidean coordinates of an object or camera undergoing general affine motion are determined for paracatadioptric imaging systems via a nonlinear observer. The nonlinear observer asymptotically determines the range information provided that the motion parameters are known. The observer is developed through a Lyapunov-based design and stability analysis, and simulation results are provided that illustrate the performance of the state estimator. The contributions of this paper are that the developed observer: 1) can be applied to both affine and nonaffine systems; 2) can be used for both the fixed and moving camera configurations; 3 ) is continuous; and 4) yields an asymptotic result.
\end{abstract}

Index Terms-Lyapunov methods, nonlinear observer, paracatadioptric vision systems, range identification, vision-based estimation.

\section{INTRODUCTION}

Conventional (i.e., planar) imaging systems are restrictive for some applications due to limitations in the field-of-view (FOV). To improve the FOV, researchers have proposed a variety of techniques including: fish-eye lenses [1], rotating imaging systems [2], cluster of cameras [3], and catadioptric systems with 3-D image surfaces (i.e., spherical, elliptical, hyperboloid, and paraboloid mirrors) [4]. The research described in this paper is focused on catadioptric systems. A catadioptric system combines reflective (catoptric) and refractive (dioptric) elements (i.e., a camera and a mirror) [5]. Catadioptric systems with a single effective viewpoint are classified as central catadioptric systems. Central catadioptric systems are desirable because they yield pure perspective images [6]. In [4], Baker and Nayar derived the complete class of single-lens single-mirror catadioptric systems (e.g., paraboloid mirror under orthographic projection) that satisfy the single viewpoint constraint.

Catadioptric systems provide a larger FOV in a manner that is favorable over alternative technologies. For example, a rotating camera system has a reduced effective bandwidth, moving parts, and extra care has to be taken to eliminate blur as the acquired images are stitched together to construct a panoramic scene. For many applications the cost of a cluster of cameras is inhibitive when compared to a catadioptric system with a similar FOV. Moreover, the viewpoints of all the cameras must coincide for a cluster of cameras to generate pure perspective images, which is a nontrivial calibration obstacle.

Catadioptric systems also exhibit several limitations. In general, the coordinates of an object are projected onto a mirror and then onto a

Manuscript received April 26, 2006; revised June 14, 2007. First published August 22, 2008; current version published September 10, 2008. This work was supported in part by NSF Career Award 0547448, NSF SGER 0738091, AFOSR Contracts F49620-03-1-0381 and F49620-03-1-0170, AFRL Contract FA4819-05-D-0011, by the Department of Energy URPR Program under Grant DE-FG04-86NE37967, and by BARD, the United States-Israel Binational Agricultural Research and Development Fund, under Grant US-3715-05. Recommended by Associate Editor J. Hespanha.

The authors are with the Department of Mechanical and Aerospace Engineering, University of Florida, Gainesville, FL 32611-6250 USA (e-mail: gqhu@ufl.edu; adarren@ufl.edu; sumitg@ufl.edu; wdixon@ufl.edu).

Color versions of one or more of the figures in this paper are available online at http://ieeexplore.ieee.org.

Digital Object Identifier 10.1109/TAC.2008.928312 camera lens. For cameras that use a lens that yields a perspective projection, the alignment of the lens and the mirror must be calibrated to account for the distance between them. Paracatadioptric systems are a special case of a central catadioptric systems that are constructed with a paraboloid mirror and an orthographic lens. The use of the orthographic lens reduces the alignment requirements, and hence, simplifies the calibration of the system, as well as the computation of pure-perspective images [7]. In comparison to other technologies that extend the FOV, another limitation of catadioptric systems is that the use of a curved mirror warps the image. This distorted image mapping can be resolved through computer vision methods, but the additional nonlinearity in the transformation presents an additional obstacle for reconstructing the Euclidean coordinates of observed feature points.

Many applications (e.g., autonomous vehicle guidance and navigation, visual servo control, etc.) require the interpretation of the Euclidean coordinates of features of a 3-D object through 2-D images. Reconstructing the Euclidean coordinates of observed feature points is a challenging problem of significant interest even for conventional planar imaging systems because range information (i.e., the distance from the imaging system to the 3-D object) is lost in the image projection. There are generally two scenarios that are of interest: a fixed camera viewing a moving target to estimate the range and/or motion information of the target (e.g., see [8], [9]), or a moving camera viewing a still scene to recover the structure of the scene and/or the motion of the camera (e.g., see [10]). For either scenario, the relative motion dynamics of the system can be written in the same form even though the motion parameters have different physical meanings. Different tools (e.g., extended Kalman filter, nonlinear observers) have been used to address the structure and/or motion recovery problem from different points of view. Some researchers (e.g., see [10]-[13]) have applied the extended Kalman filter (EKF) to address the structure/motion recovery problem. In order to use the EKF method, a priori knowledge of the noise distribution is required, and the motion recovery algorithm is developed based on the linearization of the nonlinear vision-based motion estimation problem.

Due to restrictions with linear methods, researchers have developed various nonlinear observers (e.g., see [8], [9], [14]-[16]). For example, several researchers have investigated the range identification problem for conventional imaging systems when the motion parameters are known. In [15], Jankovic and Ghosh developed a discontinuous observer, known as the identifier based observer (IBO), to exponentially identify range information of features from successive images of a camera where the object model is based on known skew-symmetric affine motion parameters. In [14], Chen and Kano generalized the object motion beyond the skew-symmetric form of [15] and developed a new discontinuous observer that exponentially forced the state observation error to be uniformly ultimately bounded (UUB). In comparison to the UUB result of [14], a continuous observer was constructed in [8] to asymptotically identify the range information for a general affine system with known motion parameters. That is, the result in [8] eliminated the skew-symmetric assumption and yielded an asymptotic result with a continuous observer. More recently, a state estimation strategy was developed in [17], [18] for affine systems with known motion parameters where only a single homogeneous observation point is provided (i.e., a single image coordinate). In [16], a reduced order observer was developed to yield a semi-global asymptotic stability result for a fixed camera viewing a moving object with known motion parameters for a pinhole camera.

Motivated by the benefits of an improved FOV, Ma et al. explored the application of the IBO of [15] to various 3-D imaging surfaces with 
a single center of projection, including the sphere, ellipsoid, and paraboloid. The results in [19] indicate that the IBO could be applied to determine range information for image dynamics that maintain an affine form such as when an object feature is projected onto a sphere or ellipsoid. However, [19] points out that, in general, the projection onto a 3-D image surface with a single center of projection may not maintain an affine form. A projection model for a paracatadioptric system was then considered in [19] that was derived based on features projecting to the surface of a paraboloid, and the fact that the projection of a 3-D point can only be observed up to a homogenous line. The specific selection of the state variables resulted in a nonaffine form that motivated $\mathrm{Ma}$ et. al. to propose a range identification method based on a sequence of linear approximation-based observers. More recently, Gupta et al. constructed a nonlinear observer in [20], based on the work in [8], to asymptotically identify the range for the nonaffine paracatadioptric system considered in [19].

The contribution of the current paper (and our preliminary work in [20], [21]) is the development of a nonlinear estimator to extract the range information (and hence, the Euclidean coordinates) from a paracatadioptric system where the image dynamics are expressed in affine or nonaffine forms. The contributions in this paper are that the developed nonlinear observer can be applied to both affine and nonaffine systems; can be applied to both the fixed and moving camera configurations; is continuous; and leads to an asymptotic result. A Lyapunov-based analysis is used to prove that the 3-D Euclidean coordinates of an object or camera moving with general affine motion are asymptotically identified provided sufficient observability conditions are satisfied. Numerical simulation results are provided to illustrate the performance of the observer.

\section{OBJECT MOTION MODEL}

\section{A. Affine Euclidean Motion}

For the development in this paper, we consider the scenario of a fixed camera viewing a moving target to estimate the range and/or motion information of the target (cf., [8], [9], [14], [16]-[19] and therein) and the scenario of a moving camera viewing a static scene to recover the structure of the scene and/or the motion of the camera (cf., [10], [22], [23], and therein). In both scenarios, the relative motion dynamics can be written in the same form, although the motion parameters have different physical meanings. Specifically, for both scenarios, the affine motion dynamics can be expressed as

$$
\left[\begin{array}{l}
\dot{x}_{1} \\
\dot{x}_{2} \\
\dot{x}_{3}
\end{array}\right]=\left[\begin{array}{lll}
a_{11} & a_{12} & a_{13} \\
a_{21} & a_{22} & a_{23} \\
a_{31} & a_{32} & a_{33}
\end{array}\right]\left[\begin{array}{l}
x_{1} \\
x_{2} \\
x_{3}
\end{array}\right]+\left[\begin{array}{l}
b_{1} \\
b_{2} \\
b_{3}
\end{array}\right]
$$

where $x(t)=\left[x_{1}(t), x_{2}(t), x_{3}(t)\right]^{T} \in \mathbb{R}^{3}$ denotes the unmeasurable Euclidean coordinates of either the moving target or the moving camera along the $X, Y$, and $Z$ axes of a camera fixed reference frame, respectively, where the $Z$ axis is colinear with the optical axis of the camera. In (1), the parameters $a_{i, j}(t) \in \mathbb{R} \forall i, j=1,2,3$ of the matrix $A(t) \in \mathbb{R}^{3 \times 3}$ and $b(t)=\left[b_{1}, b_{2}, b_{3}\right]^{T} \in \mathbb{R}^{3}$ denote the motion parameters. The affine motion dynamics introduced in (1) are expressed in a general form that describes an object motion consisting of a rotation, translation, and linear deformation [24].

Assumption 1: The motion parameters in $A(t)$ and $b(t)$ introduced in (1) are assumed to be known, bounded functions of time that are second order differentiable (cf. [8], [12], [14]-[21]).

To illustrate how the affine dynamics in (1) represent both the stationary camera moving object scenario and the moving camera stationary object scenario, consider a feature point attached to a target as in Fig. 1. For stationary camera moving object scenario, $\mathcal{F}_{c}$ denotes an inertial coordinate frame attached to the camera, and $x(t)$ (expressed

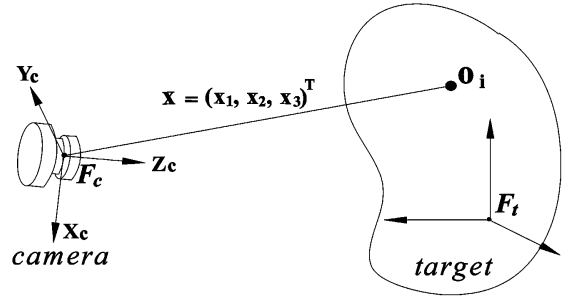

Fig. 1. Problem scenario of a camera and a target.

in $\mathcal{F}_{c}$ ) denotes the coordinates of the target feature point $O_{i}$. The time derivative of $x(t)$ can be expressed as

$$
\dot{x}=-\left[\omega_{t}\right]^{\times} x-v_{t}=A x+b
$$

where $v_{t}(t), \omega_{t}(t) \in \mathbb{R}^{3}$ denote the linear and angular velocities of target with respect to the inertial camera frame $\mathcal{F}_{c}$, respectively. Potential applications for this scenario under the restriction of Assumption 1 include examples where the object is moving with a known/measurable linear and angular velocity where the goal is to estimate the Euclidean position of the moving target in time, such as: parts moving on a conveyor belt on assembly line, vehicles on a railway or subway, air and/or ground traffic monitoring from a fixed location.

For the moving camera stationary object scenario, $\mathcal{F}_{c}$ denotes a body-fixed coordinate frame attached to the camera, and $\mathcal{F}_{t}$ denotes an inertial coordinate frame. The linear and angular velocities of the target (i.e., $v_{t}(t)$ and $\left.\omega_{t}(t)\right)$ with respect to the camera (expressed in $\mathcal{F}_{c}$ ) can be written as

$$
v_{t}=-R v_{c} \quad \omega_{t}=-R \omega_{c}
$$

where $R(t) \in \mathbb{R}^{3 \times 3}$ denotes the corresponding rotation between $\mathcal{F}_{c}$ and $\mathcal{F}_{t}$, and $v_{c}(t)$ and $\omega_{c}(t)$ denote the linear and angular velocity of the camera, respectively. Based on (3), the relationship in (2) can be rewritten as

$$
\dot{x}=\left[R \omega_{c}\right]^{\times} x+R v_{c}=A x+b .
$$

Potential applications for this scenario under the restriction of Assumption 1 include examples where the camera is moving with a known/ measurable linear and angular velocity where the goal is to estimate the Euclidean position of the moving camera in time, such as: inertial navigation in GPS denied environments and simultaneous localization and mapping (SLAM).

Remark 1: The implications of Assumption 1 for the stationary camera moving object scenario are that $v_{t}(t)$ and $\omega_{t}(t)$ must be known/measurable, and the implications of Assumption 1 for the moving camera stationary object scenario are that $v_{c}(t)$ and $\omega_{c}(t)$ must be measured along with $R(t)$.

\section{B. Projection}

The projection of the coordinates $x(t)$ onto a paraboloid mirror with its focus at the origin (see Fig. 2) can be expressed as [25]

$$
y \triangleq\left[\begin{array}{lll}
y_{1} & y_{2} & y_{3}
\end{array}\right]^{T}=\frac{2 f}{L}\left[\begin{array}{lll}
x_{1} & x_{2} & x_{3}
\end{array}\right]^{T}
$$

where $f \in \mathbb{R}$ denotes the constant known distance between the focal point and the vertex of the paraboloid, and $L(x) \in \mathbb{R}$ is defined as

$$
L \triangleq-x_{3}+\sqrt{x_{1}^{2}+x_{2}^{2}+x_{3}^{2}} .
$$

Because the projection from the paraboloid mirror to the camera is orthographic in nature (i.e., reflected light rays are parallel to the optical axis), $y_{1}(t)$ and $y_{2}(t)$ correspond to the measured pixel coordinates 




Fig. 2. Euclidean point projected onto paraboloid mirror and then reflected to an orthographic camera.

of the camera. Also, because the paraboloid is rotationally symmetric, $y_{3}(t)$ is computed from the measured pixel coordinates as

$$
y_{3}=\frac{\left(y_{1}^{2}+y_{2}^{2}\right)}{4 f}-f \text {. }
$$

To facilitate subsequent development, the auxiliary signal $y_{4}(t) \in \mathbb{R}$ is defined as

$$
y_{4} \triangleq \frac{2 f}{L}
$$

and contains the unknown range information. Substituting (8) into (5), yields the following relationship:

$$
y=y_{4} x
$$

Taking the time derivative of (9) and utilizing (1), the following PDS can be determined:

$$
\dot{y}=\Omega_{1}+g,
$$

where $\Omega_{1}\left(y_{1} y_{2}, y_{3}, t\right) \in \mathbb{R}^{3}$ denotes a vector of measurable and known signals defined as

$$
\Omega_{1} \triangleq\left[\begin{array}{cc}
\sum_{j=1}^{3}\left(a_{1 j} y_{j}+\frac{1}{2 f} a_{3 j} y_{1} y_{j}\right) \\
\sum_{j=1}^{3}\left(a_{2 j} y_{j}+\frac{1}{2 f} a_{3 j} y_{2} y_{j}\right) \\
\sum_{j=1}^{3}\left(a_{3 j} y_{j}+\frac{1}{2 f} a_{3 j} y_{3} y_{j}\right)
\end{array}\right]
$$

and the unmeasurable signal $g(t) \triangleq\left[g_{1}(t), g_{2}(t), g_{3}(t)\right] \in \mathbb{R}^{3}$ is defined as

$$
g_{i} \triangleq \Omega_{0} y_{i}+b_{i} y_{4} \quad \forall i=1,2,3,
$$

where $\Omega_{0}\left(y_{1} y_{2}, y_{3}, t\right) \in \mathbb{R}$ is defined as

$$
\Omega_{0} \triangleq \frac{b_{3}}{L}-\frac{y_{1} \dot{x}_{1}+y_{2} \dot{x}_{2}+y_{3} \dot{x}_{3}}{2 f\left(L+x_{3}\right)} .
$$

From (12), the signal $y_{4}(t)$ containing the range information can be expressed as

$$
y_{4}^{2}=\frac{\left(y_{2} g_{1}-y_{1} g_{2}\right)^{2}+\left(y_{3} g_{1}-y_{1} g_{3}\right)^{2}+\left(y_{3} g_{2}-y_{2} g_{3}\right)^{2}}{\left(y_{2} b_{1}-y_{1} b_{2}\right)^{2}+\left(y_{3} b_{1}-y_{1} b_{3}\right)^{2}+\left(y_{3} b_{2}-y_{2} b_{3}\right)^{2}} .
$$

Assumption 2: In contrast to the systems examined in [19] and [20], the development in this section is based on the more general (and more practical) assumption that the focal point is not at the vertex of the paraboloid (i.e., $f>0$ ). Moreover, the focal point is not a vanishing point (i.e., $f \in \mathcal{L}_{\infty}$ ).
Assumption 3: The image-space feature coordinates $y_{1}(t), y_{2}(t)$ are bounded functions of time; hence, (7) can be used to conclude that $y_{3}(t) \in \mathcal{L}_{\infty}$.

Assumption 4: The object feature is not a vanishing point (i.e., $L \in$ $\mathcal{L}_{\infty}$, therefore $y_{4}(t) \neq 0$ ). We assume that $L \neq 0$ (i.e., $x_{1}, x_{2} \neq 0$ simultaneously); hence, the object feature does not intersect the optical axis of the imaging system. Since $L \neq 0$, (8) can be used to conclude that $y_{4}(t) \in \mathcal{L}_{\infty}$.

Assumption 5: If $g(t)$ can be identified, then $y_{4}(t)$ can be determined from (14), provided $b_{1}, b_{2}, b_{3} \neq 0$ simultaneously. This observability assumption physically means that the object must translate in at least one direction.

Remark 2: Based on Assumptions 1-5, the expressions given in (10)-(14) can be used to determine that $\dot{y}(t), \Omega_{1}(t)$, and $g(t) \in \mathcal{L}_{\infty}$. Given that these signals are bounded, Assumptions 1-5 can be used to prove that

$$
\|g(\cdot)\| \leq \zeta_{1} \quad\|\dot{g}(\cdot)\| \leq \zeta_{2} \quad\|\ddot{g}(\cdot)\| \leq \zeta_{3}
$$

where $\zeta_{1}, \zeta_{2}$, and $\zeta_{3} \in \mathbb{R}$ denote known positive constants.

\section{RANGE IDENTIFICATION FOR AFFINE SYSTEMS}

\section{A. Objective}

The objective of this section is to extract the Euclidean coordinate information of the object feature from its projection onto the paracatadioptric system. From (9) and the fact that $y_{1}(t), y_{2}(t)$ and $y_{3}(t)$ are measurable, if $y_{4}(t)$ could be identified then the complete Euclidean coordinates of the feature can be determined. To achieve this objective, an estimator is constructed based on the unmeasurable image-space dynamics for $y(t)$. To quantify the objective, a measurable estimation error, denoted by $e(t) \triangleq\left[e_{1}(t), e_{2}(t), e_{3}(t)\right]^{T} \in \mathbb{R}^{3}$, is defined as follows:

$$
e=y-\hat{y}
$$

where $\hat{y}(t) \triangleq\left[\hat{y}_{1}(t), \hat{y}_{2}(t), \hat{y}_{3}(t)\right]^{T} \in \mathbb{R}^{3}$ denotes a subsequently designed estimate. An unmeasurable ${ }^{1}$ filtered estimation error, denoted by $r(t) \triangleq\left[r_{1}(t), r_{2}(t), r_{3}(t)\right]^{T} \in \mathbb{R}^{3}$, is also defined as

$$
r=\dot{e}+\alpha e
$$

where $\alpha \in \mathbb{R}^{3 \times 3}$ denotes a diagonal matrix of positive constant gains $\alpha_{1}, \alpha_{2}, \alpha_{3} \in \mathbb{R}$. Motivation for the development of the filtered estimation error in (17), is that the subsequent observer is based on the PDS given in (10). If $g(t)$ in (10) can be identified, the fact that the feature point coordinates $y_{i}(t) \forall i=1,2,3$ are measurable can be used along with (14) to compute $y_{4}(t)$ provided the observability condition in Assumption 5 is satisfied.

\section{B. Estimator Design and Error System}

Based on (10) and the subsequent analysis, the following estimation signals are defined:

$$
\dot{\hat{y}}=\Omega_{1}+\hat{g}
$$

where $\hat{g}(t) \triangleq\left[\hat{g}_{1}(t), \hat{g}_{2}(t), \hat{g}_{3}(t)\right]^{T} \in \mathbb{R}^{3}$ denotes a subsequently designed estimate for $g(t)$. The following error dynamics are obtained after taking the time derivative of $e(t)$ and utilizing (10) and (18):

$$
\dot{e}=g-\hat{g} \text {. }
$$

${ }^{1}$ The filtered estimation signal is unmeasurable due to a dependence on the unmeasurable terms $g_{1}(t), g_{2}(t), g_{3}(t)$. 
Based on the structure of (17) and (19), $\dot{\hat{g}}(t)$ is designed as follows [8]:

$$
\dot{\hat{g}}=-\left(k_{s}+\alpha\right) \hat{g}+\gamma \operatorname{sgn}(e)+\alpha k_{s} e
$$

where $k_{s}, \gamma \in \mathbb{R}^{3 \times 3}$ denote diagonal matrices of positive constant estimation gains, and the notation $\operatorname{sgn}(\cdot)$ is used to indicate a vector with the standard signum function applied to each element of the argument. The structure of the estimator in (20) contains discontinuous terms; however, as discussed in [8], the overall structure of the estimator is continuous (i.e., $\hat{g}(t)$ is continuous). After using (17), (19), and (20), the following expression can be obtained:

$$
\dot{r}=\eta-k_{s} r-\gamma \operatorname{sgn}(e)
$$

where $\eta(t) \triangleq\left[\begin{array}{lll}\eta_{1} & \eta_{2} & \eta_{3}\end{array}\right]^{T} \in \mathbb{R}^{3}$ is defined as

$$
\eta=\dot{g}+\left(k_{s}+\alpha\right) g
$$

Based on (15) and (22), the following inequalities can be developed:

$$
|\eta(\cdot)| \leq \zeta_{4} \quad|\dot{\eta}(\cdot)| \leq \zeta_{5}
$$

where $\zeta_{4}$ and $\zeta_{5} \in \mathbb{R}$ denote known positive constants.

Remark 3: Considering (14), the unmeasurable signal $y_{4}(t)$ can be identified if $\hat{g}(t)$ approaches $g(t)$ as $t \rightarrow \infty$ (i.e., $\hat{y}_{1}(t), \hat{y}_{2}(t)$ and $\hat{y}_{3}(t)$ approach $y_{1}(t), y_{2}(t)$ and $y_{3}(t)$ as $\left.t \rightarrow \infty\right)$ since the parameters $b_{i}(t) \forall i=1,2,3$ are assumed to be known, and $y_{1}(t), y_{2}(t)$ and $y_{3}(t)$ are measurable. After $y_{4}(t)$ is identified, (9) can be used to extract the 3-D Euclidean coordinates of the object feature (i.e., determine the range information). To prove that $\hat{g}(t)$ approaches $g(t)$ as $t \rightarrow \infty$, the subsequent development will focus on proving that $\|\dot{e}(t)\| \rightarrow 0$ and $\|e(t)\| \rightarrow 0$ as $t \rightarrow \infty$ based on (16) and (19).

\section{ANALYSIS}

The following theorem and associated proof can be used to conclude that the observer design of (18) and (20) can be used to identify the unmeasurable signal $y_{4}(t)$.

Theorem 1: For the paracatadioptric system in (10)-(13), the unmeasurable signal $y_{4}(t)$ (and hence, the Euclidean coordinates of the object feature) can be asymptotically determined from the estimator in (18) and (20) provided the elements of the constant diagonal matrix $\gamma$ introduced in (20) are selected according to the sufficient condition

$$
\gamma_{i} \geq \zeta_{4}+\frac{1}{\alpha_{i}} \zeta_{5}
$$

$\forall i=1,2,3$, where $\zeta_{4}$ and $\zeta_{5}$ are defined in (23).

Proof: Consider a non-negative function $V(t) \in \mathbb{R}$ as follows (i.e., a Lyapunov function candidate):

$$
V=\frac{1}{2} r^{T} r
$$

After taking the time derivative of (25) and substituting for the error system dynamics given in (21), the following expression can be obtained:

$$
\dot{V}=-r^{T} k_{s} r+(\dot{e}+\alpha e)^{T}(\eta-\gamma \operatorname{sgn}(e)) .
$$

After integrating (26) and exploiting the fact that

$$
\xi_{i} \cdot \operatorname{sgn}\left(\xi_{i}\right)=\left|\xi_{i}\right| \forall \xi_{i} \in \mathbb{R}
$$

the following inequality can be obtained:

$$
\begin{aligned}
V(t) \leq V\left(t_{0}\right)- & \int_{t_{0}}^{t}\left(r^{T}(\sigma) k_{s} r(\sigma)\right) d \sigma \\
& +\sum_{i=1}^{3} \alpha_{i} \int_{t_{0}}^{t}\left|e_{i}(\sigma)\right|\left(\left|\eta_{i}(\sigma)\right|-\gamma_{i}\right) d \sigma+\chi_{i}
\end{aligned}
$$

where the auxiliary terms $\chi_{i}(t) \in \mathbb{R}$ are defined as

$$
\chi_{i}=\int_{t_{0}}^{t} \dot{e}_{i}(\sigma) \eta_{i}(\sigma) d \sigma-\gamma_{i} \int_{t_{0}}^{t} \dot{e}_{i}(\sigma) \operatorname{sgn}\left(e_{i}(\sigma)\right) d \sigma
$$

$\forall i=1,2,3$. The integral expression in (28) can be evaluated as

$$
\begin{aligned}
\chi_{i}= & \left.e_{i}(\sigma) \eta_{i}(\sigma)\right|_{t_{0}} ^{t} \\
& -\int_{t_{0}}^{t} e_{i}(\sigma) \dot{\eta}_{i}(\sigma) d \sigma-\left.\gamma_{i}\left|e_{i}(\sigma)\right|\right|_{t_{0}} ^{t} \\
= & e_{i}(t) \eta_{i}(t)-\int_{t_{0}}^{t} e_{i}(\sigma) \dot{\eta}_{i}(\sigma) d \sigma-\gamma_{i}\left|e_{i}(t)\right| \\
& -e_{i}\left(t_{0}\right) \eta_{i}\left(t_{0}\right)+\gamma_{i}\left|e_{i}\left(t_{0}\right)\right|
\end{aligned}
$$

$\forall i=1,2,3$. Substituting (29) into (27) and performing some algebraic manipulation yields

$$
V(t) \leq V\left(t_{0}\right)-\int_{t_{0}}^{t}\left(r^{T}(\sigma) k_{s} r(\sigma)\right) d \sigma+\chi_{4}+\zeta_{0}
$$

where the auxiliary terms $\chi_{4}(t), \zeta_{0} \in \mathbb{R}$ are defined as

$$
\begin{aligned}
\chi_{4}= & \sum_{i=1}^{3} \alpha_{i} \int_{t_{0}}^{t}\left|e_{i}(\sigma)\right|\left(\left|\eta_{i}(\sigma)\right|+\frac{1}{\alpha_{i}}\left|\dot{\eta}_{i}(\sigma)\right|-\gamma_{i}\right) d \sigma \\
& +\sum_{i=1}^{3}\left|e_{i}(t)\right|\left(\left|\eta_{i}(t)\right|-\gamma_{i}\right) \\
\zeta_{0}= & \sum_{i=1}^{3}-e_{i}\left(t_{0}\right) \eta_{i}\left(t_{0}\right)+\gamma_{i}\left|e_{i}\left(t_{0}\right)\right| .
\end{aligned}
$$

Provided $\gamma_{i} \forall i=1,2,3$ are selected according to the inequality introduced in (24), $\chi_{4}(t)$ will always be negative or zero; hence, $V(t)$ can be upper bounded as

$$
V(t) \leq V\left(t_{0}\right)-\int_{t_{0}}^{t}\left(r^{T}(\sigma) k_{s} r(\sigma)\right) d \sigma+\zeta_{0} .
$$

From (25) and (30), the following inequalities can be determined:

$$
V\left(t_{0}\right)+\zeta_{0} \geq V(t) \geq 0
$$

hence, $r(t) \in \mathcal{L}_{\infty}$. The expression in (30) can be used to determine that

$$
\int_{t_{0}}^{t}\left(r^{T}(\sigma) k_{s} r(\sigma)\right) d \sigma \leq V\left(t_{0}\right)+\zeta_{0}<\infty .
$$

By definition, (31) can now be used to prove that $r(t) \in \mathcal{L}_{2}$. From the fact that $r(t) \in \mathcal{L}_{\infty},(16)$ and (17) can be used to prove that $e(t), \dot{e}(t)$, $\hat{y}(t)$, and $\dot{\hat{y}}(t) \in \mathcal{L}_{\infty}$. The expressions in (18) and (20) can be used to determine that $\hat{g}(t)$ and $\dot{\hat{g}}(t) \in \mathcal{L}_{\infty}$. Based on (15), the expressions in (21) and (22) can be used to prove that $\eta(t), \dot{\eta}(t), \dot{r}(t) \in \mathcal{L}_{\infty}$. Based on the fact that $r(t), \dot{r}(t) \in \mathcal{L}_{\infty}$ and that $r(t) \in \mathcal{L}_{2}$, Barbalat's Lemma [26] can be used to prove that $\|r(t)\| \rightarrow 0$ as $t \rightarrow \infty$; hence, standard linear analysis can be used to prove that $\|e(t)\| \rightarrow 0$ and $\|\dot{e}(t)\| \rightarrow 0$ as $t \rightarrow \infty$. Based on the fact that $\|e(t)\| \rightarrow 0$ and $\|\dot{e}(t)\| \rightarrow 0$ as $t \rightarrow \infty$, the expression given in (16) can be used to determine that $\hat{y}_{1}(t), \hat{y}_{2}(t)$ and $\hat{y}_{3}(t)$ approach $y_{1}(t), y_{2}(t)$ and $y_{3}(t)$ as $t \rightarrow \infty$, respectively. Therefore, the expression in (19) can be used to determine that $\hat{g}(t)$ approaches $g(t)$ as $t \rightarrow \infty$. The result that $\hat{g}(t)$ approaches $g(t)$ as $t \rightarrow \infty$, the fact that the parameters $b_{i}(t) \forall i=1,2,3$ are assumed to be known, and the fact that the image-space signals $y_{1}(t), y_{2}(t)$ and $y_{3}(t)$ are measurable can be used to identify the unknown signal $y_{4}(t)$ 
from (14). Once $y_{4}(t)$ is identified, the complete Euclidean coordinates of the object feature can be determined using (9).

\section{RANGE IDENTIFICATION FOR NONAFFINE SYSTEMS}

As pointed out by [19], the image projection from a general 3-D surface with a single center of projection may not maintain an affine form in general. A contribution of the observer proposed in this paper (along with our preliminary work in [20], [21]) is that it can be used to extract the range information for both affine and nonaffine dynamics. To illustrate this point, we consider the paracatadioptric example provided in [19]. In [19], the projection of an object feature $x(t)$ onto a paraboloid mirror is expressed as

$$
\left[\begin{array}{llll}
y_{1} & y_{2} & y_{3} & y_{4}
\end{array}\right]^{T}=\frac{1}{x_{1}^{2}+x_{2}^{2}}\left[\begin{array}{llll}
x_{1} x_{3} & x_{2} x_{3} & x_{3}^{2} & 1
\end{array}\right]^{T}
$$

where $y_{3}(t)$ can be computed from the measured pixel coordinates as

$$
y_{3}=y_{1}^{2}+y_{2}^{2} \text {. }
$$

The time derivative of (32) yields

$$
\left[\begin{array}{lll}
\dot{y}_{1} & \dot{y}_{2} & \dot{y}_{3}
\end{array}\right]^{T}=\Omega_{3}+\bar{g}
$$

where $\Omega_{3}\left(y_{1}, y_{2}, y_{3}, t\right) \in \mathbb{R}^{3}$ denotes a vector of measurable and known signals defined as

$$
\Omega_{3} \triangleq\left[\begin{array}{c}
\sum_{j=1}^{3}\left\{a_{1 j} y_{j}+\frac{y_{1} y_{j}}{y_{3}}\left[a_{3 j}-2\left(a_{1 j} y_{1}+a_{2 j} y_{2}\right)\right]\right\} \\
\sum_{j=1}^{3}\left\{a_{2 j} y_{j}+\frac{y_{2} y_{j}}{y_{3}}\left[a_{3 j}-2\left(a_{1 j} y_{1}+a_{2 j} y_{2}\right)\right]\right\} \\
\sum_{j=1}^{3}\left\{a_{3 j} y_{j}+y_{j}\left[a_{3 j}-2\left(a_{1 j} y_{1}+a_{2 j} y_{2}\right)\right]\right\}
\end{array}\right]
$$

and the unmeasurable signal $\bar{g}(t) \triangleq\left[\bar{g}_{1}(t), \bar{g}_{2}(t), \bar{g}_{3}(t)\right]^{T} \in \mathbb{R}^{3}$ is defined as

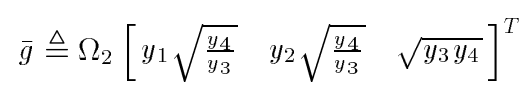

where $\Omega_{2}\left(y_{1}, y_{2}, y_{3}, t\right) \in \mathbb{R}^{3 \times 3}$ is defined as

$$
\Omega_{2} \triangleq\left[\begin{array}{ccc}
\left(b_{3}-2 b_{1} y_{1}\right) & -2 b_{2} y_{1} & b_{1} \\
-2 b_{1} y_{2} & \left(b_{3}-2 b_{2} y_{2}\right) & b_{2} \\
-2 b_{1} y_{3} & -2 b_{2} y_{3} & 2 b_{3}
\end{array}\right]
$$

Based on the particular selection of $y_{4}(t)$ in (32), the projected dynamics in (34)-(37) are in a nonaffine form. Since the projective relationship in this section is different from that in Section III, the observability conditions in Assumption 5 need to be modified as in Assumption 6.

Assumption 6: The matrix $\Omega_{2}\left(y_{1}, y_{2}, y_{3}, t\right)$ introduced in (37) is invertible provided

$$
b_{3} \neq 0
$$

and

$$
b_{3}^{2}-2 b_{2} b_{3} y_{2}+b_{2}^{2} y_{3}-2 b_{1} b_{3} y_{1}+b_{1}^{2} y_{3} \neq 0 \text {. }
$$

After utilizing (33), the condition in (39) can be written as

$$
\left(y_{1}-k_{1}\right)^{2}+\left(y_{2}-k_{2}\right)^{2} \neq 0
$$

where $k_{1}(t)$ and $k_{2}(t) \in \mathbb{R}$ are auxiliary terms defined as

$$
k_{1}=\frac{b_{1} b_{3}}{b_{1}^{2}+b_{2}^{2}} \quad k_{2}=\frac{b_{2} b_{3}}{b_{1}^{2}+b_{2}^{2}} .
$$

Geometrically, the observability condition in (40) indicates that the projection of the object feature cannot intersect the point $\left(k_{1}, k_{2}\right)$. For the special case when $b_{1}=b_{2}=0$, (39) reduces to (38). If the conditions in (38) and (40) are violated, then the range to the feature point can not be determined because the feature point is projected to the mirror tip (i.e., $y_{1}=y_{2}=0$ ), which is a known point of ambiguity. It is interesting to note that in most commercial paracatadioptric systems, the mirror is mounted to the camera lens in such a manner that the mirror tip is covered.

Based on (32) and the fact that $y_{1}(t), y_{2}(t)$ and $y_{3}(t)$ are measurable, if $y_{4}(t)$ could be identified then the complete Euclidean coordinates of the feature can be determined. From (36), it is clear that identifying $\bar{g}(t)$ is equivalent to identifying $y_{4}(t)$ provided the observability conditions in Assumption 6 are satisfied. Comparisons between (10) and (34) indicate that both PDS can be written in the same form; hence, the observer in (18) and (20) can also be applied to the nonaffine system in (34)-(37)

\section{NUMERICAL SIMULATION}

In this section, numerical simulation results are provided to illustrate the performance of the range identification observer for the paracatadioptric system. The object feature is assigned the following affine motion dynamics [8]:

$$
\left[\begin{array}{l}
\dot{x}_{1} \\
\dot{x}_{2} \\
\dot{x}_{3}
\end{array}\right]=\left[\begin{array}{ccc}
-0.2 & 0.4 & -0.6 \\
0.1 & -0.2 & 0.3 \\
0.3 & -0.4 & 0.4
\end{array}\right]\left[\begin{array}{l}
x_{1} \\
x_{2} \\
x_{3}
\end{array}\right]+\left[\begin{array}{lll}
0.5 & 0.25 & 0.3
\end{array}\right]^{T}
$$

with the initial Euclidean coordinates

$$
\left[\begin{array}{lll}
x_{1}(0) & x_{2}(0) & x_{3}(0)
\end{array}\right]^{T}=\left[\begin{array}{lll}
0.4 & 0.6 & 1
\end{array}\right]^{T} .
$$

By arbitrarily letting $f=1 / 2$, the expressions in (5), (6), and (8) can be used to determine that

$$
\begin{array}{cc}
\hat{y}_{1}\left(t_{0}\right)=y_{1}\left(t_{0}\right)=1.72 & \hat{y}_{3}\left(t_{0}\right)=y_{3}\left(t_{0}\right)=4.29 \\
\hat{y}_{2}\left(t_{0}\right)=y_{2}\left(t_{0}\right)=2.58 & y_{4}\left(t_{0}\right)=4.29
\end{array}
$$

The estimates for $g(x)$ were initialized as follows:

$$
\hat{g}_{1}\left(t_{0}\right)=1 \quad \hat{g}_{2}\left(t_{0}\right)=1 \quad \hat{g}_{3}\left(t_{0}\right)=1 .
$$

After adjusting the observer gains as

$$
\begin{aligned}
k_{s} & =\operatorname{diag}\{50,50,50\} \quad \alpha=\operatorname{diag}\{15,15,15\} \\
\gamma & =\operatorname{diag}\{1,1,1\} \times 10^{-5}
\end{aligned}
$$

the resulting mismatch between $g_{1}(t)$ and $\hat{g}_{1}(t), g_{2}(t)$ and $\hat{g}_{2}(t)$, and $g_{3}(t)$ and $\hat{g}_{3}(t)$ (i.e., $\dot{e}_{1}(t), \dot{e}_{2}(t)$, and $\dot{e}_{3}(t)$ respectively) is depicted in Fig. 3. The mismatch between $y_{4}(t)$ and $\hat{y}_{4}(t)$ is provided in Fig. 4. The $y_{4}(t)$ term is obtained from numerical integration of the $\dot{y}_{4}(t)$ term, while the estimated value is obtained by replacing $g(t)$ with $\hat{g}(t)$ in (14).

Additive-white-Gaussian-noise (AWGN) was injected into the measurable image-space signals $y_{1}(t), y_{2}(t)$ via the $\operatorname{awgn}()$ function in MATLAB, while maintaining a constant signal-to-noise-ratio of 20 . 


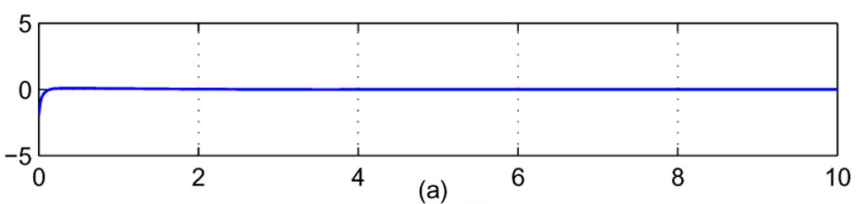

(a)

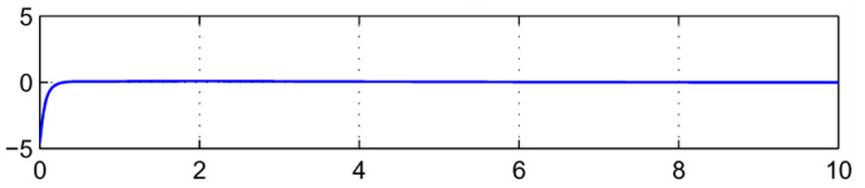

(b)

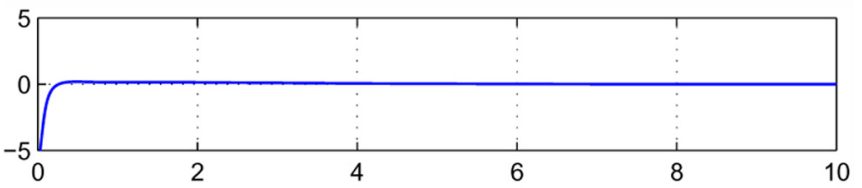

(c)

Time $[\mathrm{sec}]$

Fig. 3. Estimation error of auxiliary signals: (a) $\dot{e}_{1}(t)$, (b) $\dot{e}_{2}(t)$, and (c) $\dot{e}_{3}(t)$ in [pixels/sec].

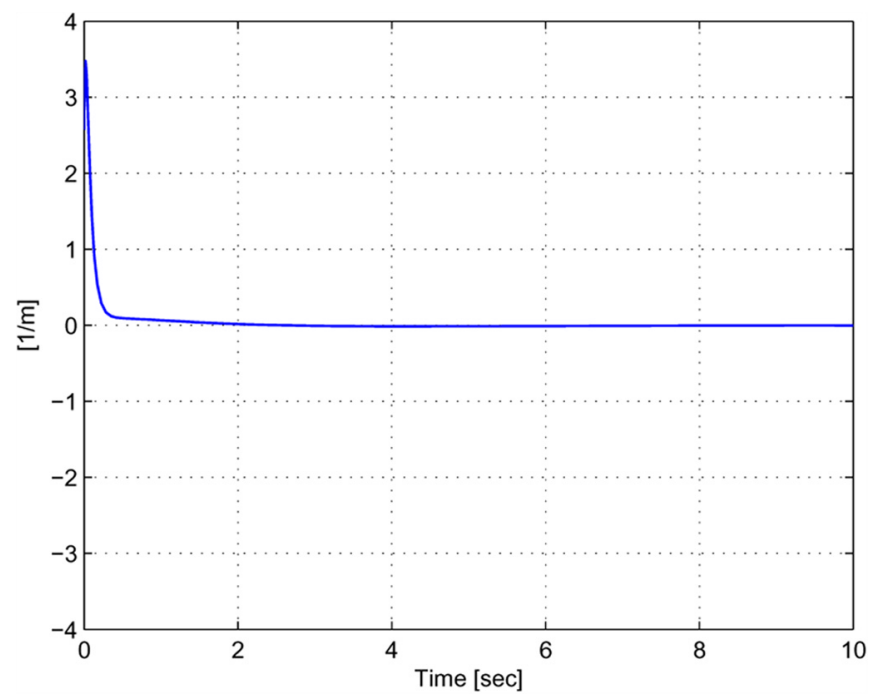

Fig. 4. Mismatch between $y_{4}(t)$ and $\hat{y}_{4}(t)$.

Without changing any of the other simulation parameters, the mismatch between $g_{1}(t)$ and $\hat{g}_{1}(t), g_{2}(t)$ and $\hat{g}_{2}(t)$, and $g_{3}(t)$ and $\hat{g}_{3}(t)$ (i.e., $\dot{e}_{1}(t), \dot{e}_{2}(t)$, and $\dot{e}_{3}(t)$, respectively) is provided in Fig. 5, while the mismatch between $y_{4}(t)$ and $\hat{y}_{4}(t)$ is provided in Fig. 6 .

The results depicted in Figs. 3-6 indicate that the proposed observer can be used to identify range, and hence, the Euclidean coordinates of an object feature moving with affine motion dynamics projected onto a paracatadioptric system provided the observability conditions are satisfied. These results are comparable to the results obtained in [8] for a planar image surface.

\section{CONCLUSION}

The results in this paper focus on the use of a nonlinear estimator to determine the range and the Euclidean coordinates of an object feature undergoing general affine motion. Motivated by the benefits of an improved FOV, the object motion is obtained from measurements by a paracatadioptric system. The nonlinear estimator is proven, via a Lyapunov-based analysis, and numerically demonstrated to asymptotically determine the range information for a paracatadioptric system

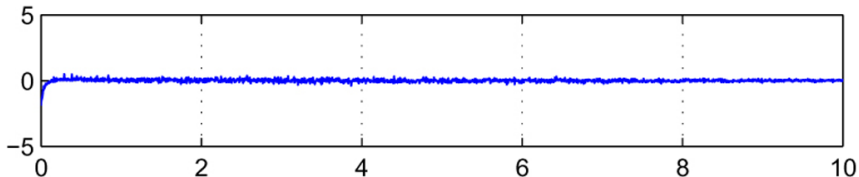

(a)

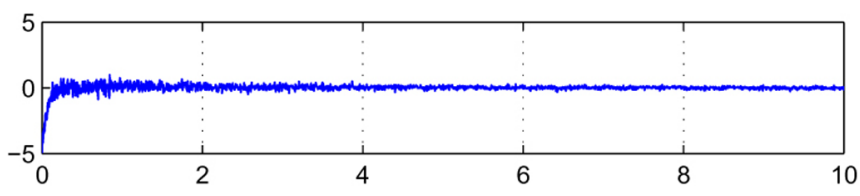

(b)



(c)

Time [sec]

Fig. 5. Estimation error of auxiliary signals in the presence of noise: (a) $\dot{e}_{1}(t)$, (b) $\dot{e}_{2}(t)$, and (c) $\dot{e}_{3}(t)$ in [pixels/sec].

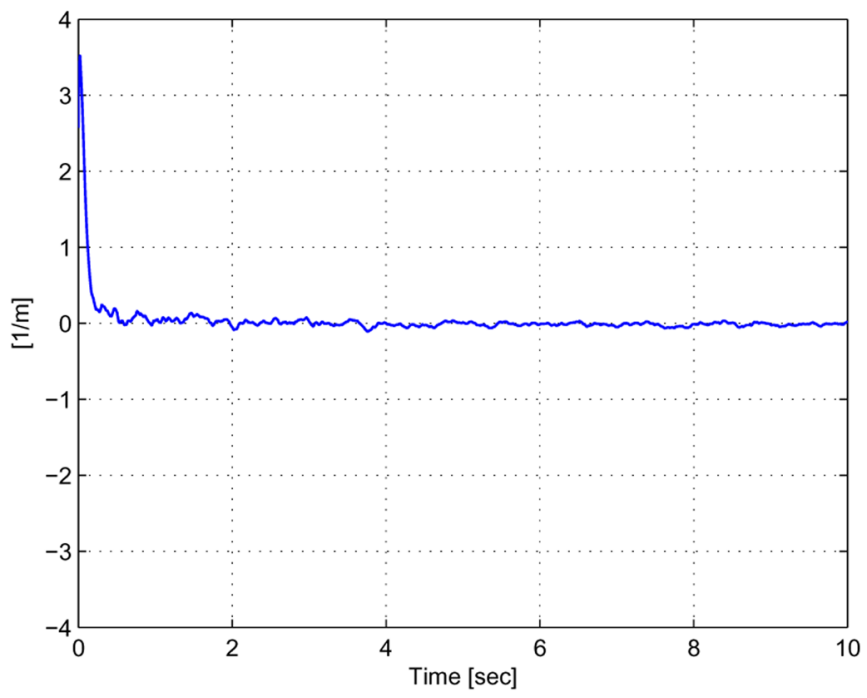

Fig. 6. Mismatch between $y_{4}(t)$ and $\hat{y}_{4}(t)$ in the presence of noise.

with known motion parameters. If specific observability conditions are satisfied, the identified range can be used to reconstruct the Euclidean coordinates of the moving object or camera.

\section{REFERENCES}

[1] Y. Xiong and K. Turkowski, "Creating image-based virtual reality using a self-calibrating fisheye lens," in Proc. IEEE Conf. Computer Vision and Pattern Recognition, 1997, pp. 237-243.

[2] M. Aggarwal and N. Ahuja, "High dynamic range panoramic imaging," in Proc. IEEE Int. Conf. Computer Vision, 2001, pp. 2-9.

[3] R. Swaminathan and S. Nayar, "Nonmetric calibration of wide-angle lenses and polycameras," IEEE Trans. Pattern Anal. Mach. Intell., vol. 22 , no. 10 , pp. $1172-1178$, Oct. 2000.

[4] S. Baker and S. Nayar, "A theory of single-viewpoint catadioptric image formation," Int. J. Comput. Vis., vol. 35, pp. 175-196, 1999.

[5] E. Hecht and A. Zadac, Optics, 3rd ed. Reading, MA: Addison-Wesley, 1997.

[6] C. Geyer and K. Daniilidis, "Catadioptric projective geometry," Int. J. Comput. Vis., vol. 45, no. 3, pp. 223-243, 2001 
[7] S. Nayar, "Catadioptric omnidirectional camera," in Proc. IEEE Conf. Computer Vision Pattern Recognition, 1997, pp. 482-488.

[8] W. E. Dixon, Y. Fang, D. M. Dawson, and T. J. Flynn, "Range identification for perspective vision systems," IEEE Trans. Autom. Control, vol. 48, no. 12, pp. 2232-2238, Dec. 2003.

[9] X. Chen and H. Kano, "State observer for a class of nonlinear systems and its application to machine vision," IEEE Trans. Autom. Control, vol. 49, no. 11, pp. 2085-2091, Nov. 2004.

[10] S. Soatto, R. Frezza, and P. Perona, "Motion estimation via dynamic vision," IEEE Trans. Autom. Control, vol. 41, no. 3, pp. 393-413, Mar. 1996.

[11] A. Chiuso, P. Favaro, H. Jin, and S. Soatto, "Structure from motion causally integrated over time," IEEE Trans. Pattern Anal. Mach. Intell., vol. 24, no. 4, pp. 523-535, Apr. 2002.

[12] H. Kano, B. K. Ghosh, and H. Kanai, "Single camera based motion and shape estimation using extended Kalman filtering," Math. Comput. Model., vol. 34, pp. 511-525, 2001.

[13] L. Matthies, T. Kanade, and R. Szeliski, "Kalman filter-based algorithm for estimating depth from image sequence," Int. J. Comput. Vis., vol. 3, pp. 209-236, 1989.

[14] X. Chen and H. Kano, "A new state observer for perspective systems," IEEE Trans. Autom. Control, vol. 47, no. 4, pp. 658-663, Apr. 2002.

[15] M. Jankovic and B. K. Ghosh, "Visually guided ranging from observations of points, lines and curves via an identifier based nonlinear observer," Syst. Control Lett., vol. 25, pp. 63-73, 1995.

[16] D. Karagiannis and A. Astolfi, "A new solution to the problem of range identification in perspective vision systems," IEEE Trans. Autom. Control, vol. 50, no. 12, pp. 2074-2077, Dec. 2005.

[17] L. Ma, Y. Chen, and K. L. Moore, "Range identification for perspective dynamic system with single homogeneous observation," in Proc. IEEE Int. Conf. Robotics and Automation, 2004, pp. 5207-5212.

[18] L. Ma, Y. Chen, and K. L. Moore, "Range identification for perspective dynamic system using linear approximation," in Proc. IEEE Int. Conf. Robotics and Automation, 2004, pp. 1658-1663.

[19] L. Ma, Y. Chen, and K. L. Moore, "Range identification for perspective dynamic systems with 3D imaging surfaces," in Proc. IEEE American Control Conf., 2005, pp. 3671-3675.

[20] S. Gupta, D. Aiken, G. Hu, and W. E. Dixon, "Lyapunov-based range and motion identification for a nonaffine perspective dynamic system," in Proc. IEEE American Control Conf., 2006, pp. 4471-4476.

[21] D. Aiken, S. Gupta, G. Hu, and W. E. Dixon, "Lyapunov-based range identification for a paracatadioptric system," in Proc. IEEE Conf. Decision Control, 2006, pp. 3879-3884.

[22] S. Soatto and P. Perona, "Reducing structure from motion: A general framework for dynamic vision part 1: Modeling," IEEE Trans. Pattern Anal. Mach. Intell., vol. 20, no. 9, pp. 933-942, Sep. 1998.

[23] S. Soatto and P. Perona, "Reducing structure from motion: A general framework for dynamic vision part 2: Implementation and experimental assessment," IEEE Trans. Pattern Anal. Mach. Intell., vol. 20, no. 9, pp. 943-960, Sep. 1998.
[24] R. Y. Tsai and T. S. Huang, "Estimating three-dimensional motion parameters of a rigid planar patch," IEEE Trans. Acoust., Speech, Signal Process., vol. ASSP-29, no. 6, pp. 1147-1152, Dec. 1981.

[25] C. Geyer and K. Danilidis, "A unifying theory for central panoramic systems and practical implications," in Proc. European Conf. Computer Vision, 2000, pp. 445-461.

[26] J. J. Slotine and W. Li, Applied Nonlinear Control. Englewood Cliffs, NJ: Prentice-Hall, 1991.

\section{Author's Reply}

Serdar E. Hamamci

I would like to thank Prof. Hohenbichler for his comments, as he reminded me of some missing references for defining the IRB and deriving Equation (8) in my paper [1] (page 1965, second column, third paragraph) and for providing me with a good opportunity to correct this case. Because I forgot to cite [2] and [3] in my paper and I have not recognized this deficiency during the review and publication process, I apologize to Prof. Hohenbichler and Prof. Ackermann.

\section{REFERENCES}

[1] S. E. Hamamci, "An algorithm for stabilization of fractional-order timedelay systems using fractional-order PID controllers," IEEE Trans. Automat. Control, vol. 52, no. 10, pp. 1964-1969, Oct. 2007.

[2] N. Hohenbichler and J. Ackermann, "Synthesis of robust PID controllers for time delay systems," in Proc. Eur. Control Conf. (ECC'03), Cambridge, MA, Sep. 1-4, 2003.

[3] N. Hohenbichler and J. Ackermann, "Computing stable regions inparameter spaces for a class of quasipolynomials," in Proc. IFAC Workshop Time Delay Syst. (TDS'03), Roquencourt, France, Sep. $8-10,2003$.

Manuscript received March 27, 2008. Current version published September 10, 2008.

The author is with the Electrical-Electronics Engineering Department, Inonu University, Malatya CO 44280, Turkey (e-mail: shamamci@inonu.edu.tr).

Digital Object Identifier 10.1109/TAC.2008.928118 